\title{
El Farol Bar problem, Potluck problem and electric energy balancing - on the importance of communication
}

\author{
Weronika Radziszewska \\ Systems Research Institute \\ Polish Academy of Sciences \\ Warsaw, Poland \\ Email:Weronika. \\ Radziszewska@ibspan.waw.pl
}

\author{
Ryszard Kowalczyk \\ Swinburne University of Technology \\ Melbourne, Australia \\ Email: rkowalczyk@swin.edu.au
}

\author{
Zbigniew Nahorski \\ Systems Research Institute \\ Polish Academy of Sciences \\ Warsaw, Poland \\ Email: Zbigniew. \\ Nahorski@ibspan.waw.pl
}

\begin{abstract}
Power balancing is an important issue when microgrids in island mode are considered. It requires active, real-time decision making to minimise the imbalances. El Farol Bar and Potluck problems are artificial problems designed to challenge the decision making process in a situation of limited information. They are theoretical, ill-defined problems designed to show that rational decision making in a situation of scarce information can give worse results than non-rational behavior. Potluck problem can be compared to electric power balancing in microgrids. But due to reduced complexity and constraints of theoretical problems and differences in goal functions the approach has to be in each case different. The study of these differences in this paper shows the importance of exchange of information between participants (or agents); it also suggest what type of information are necessary in what conditions. The amount of information that needs to be exchanged depends on the relation between participants (agents): the less cooperating their relation is, the less information they are willing to exchange.
\end{abstract}

\section{INTRODUCTION}

$\mathbf{T}$ HE POWER grids enter a new era, where the ecological impact and power security are playing an important role in their development. The introduction of relatively cheap micro sources has created a concept of microgrids: a localized part of the power network that usually is equipped with micro power sources and can be disconnected from the external power grid (operate in island mode). With new technologies, new challenges appeared, and among them management of power in the microgids. This task is difficult due to a relatively large changeability of production and consumption of power in small scale grids. The problem of power balancing became especially important for the development of microgrids operating in island mode. In such case, imbalances can lead to an ineffective system, to waste of power or even to damages of the devices. This problem is a subject of many research, where various methods have been developed for balancing and for minimizing the imbalances.

The power management can focus on the consumption side, and is called the demand side management [1] or demand response [2]. The idea behind it is to convince the power users to adjust their behavior patterns according to the energy situation in the grid or to allow some information system to do it automatically. An alternative is the supply side management, which requires managing the operating point of power sources, including switching on and off power production devices, to compensate for non-controllable production from renewable power sources. In this work, only supply side management will be considered.

Enumula and Rao described the Potluck problem in [3] and pointed out its similarity to the balancing of electric power. Both problems deal with equalizing supply and demand in an iterated way, where the supply can be only approximately assessed and balancing depends on decisions of many suppliers. The theoretical Potluck problem is claimed to be a generalization of El Farol Bar problem described in [4], where it is shown that, when there is lack of information, the inductive reasoning gives better results than rational reasoning. The theoretical Potluck problem is very constrained and does not allow any flow of information between participants, which make the problem ill-defined. An inductive reasoning based algorithm provides an acceptable solution to the Potluck problem, in the sense that the solution oscillates around the desired outcome. For energy balancing, such a solution is not good enough: it implies that the system is constantly experiencing imbalances. Relaxing the constraints of the Potluck problem allows for a more exact solution to be found using a rational method; the analysis of which constraints need to be relaxed in the Potluck problem provide valuable information to develop a scheme in which balancing in a microgrid can be solved.

In this article, a comparison of the balancing problem, the El Farol Bar problem and Potluck problem is presented. Although it is claimed that they are similar, there are major differences that require different approach to each of them. In section II a description of the power balancing and its importance is presented. Section III explains the theoretical El Farol and Potluck problems. In section IV a comparison of the problems is presented. Section V deals with real life approaches to the power balancing issue. The final section VI concludes the paper. 


\section{BALANCING ELECTRIC ENERGY IN MICROGRIDS}

High voltage grids, unlike low-voltage microgrids, have different properties. Large grids require an approach where power flow dynamics are considered, as the power in large grids have to travel large distances and the power loses are significant. Such networks have also much more inertia and delays in changing the operation levels. When a node of such grid is not balanced (there is either an overproduction or a deficit of power) it affects all connected nodes and a number of nodes have to compensate, as the power disturbance can travel through the network, even to distant nodes. Microgrids are smaller and react faster to control requests, so they are manageable in an easier way, without considering the effect of distance, induction of power in the grid or power losses.

A microgrid is a part of an electric grid that potentially can be disconnected. Very often, microgrids are equipped with power sources (such as e.g. gas microturbines, micro wind turbines, photovoltaic panels) or power storage (e.g. batteries, flywheels) and can work in an island mode. In the island mode operation, in a microgrid it is necessary to balance the production and consumption of power to maintain the quality factors of the electric current; these guarantee the safety of the devices in the microgrid. A symbolic picture of microgrid elements is presented in Fig. 1. If a microgrid has an abundance of power production, then energy has to be wasted and/or production limited; in case of shortage of energy, some of the consuming devices should be switched off according to importance or preference. The difficulty is that a decision has to be made and it should follow the constantly changing conditions in the grid. The faster the decision is made, the less power is wasted and the safer the devices are. Power produced by some renewable sources (especially micro sources, which might lack the ability to manage their operating point) fluctuates dynamically due to sudden changes in e.g. wind and solar irradiance. Predictors, to some extent, can forecast the production and help minimizing the imbalances, but the predictions are not perfect. Consumption of energy is also very changeable and often unpredictable, especially in small microgrids, where a single device can make a noticeable difference in overall power usage. That means that the actions of a single human being can make a noticeable disruption from a typical daily power usage profile.

Balancing should make the amount produced $\left(s\left(t_{k}\right)=\right.$ $\left.\int s(t) d t\right)$, for a given time $\left(t_{k}\right)$, equal to the amount that $t \in t_{k}$ can be consumed $\left(d\left(t_{k}\right)=\int_{t \in t_{k}} d(t) d t\right)$ at that time. The real energy balancing is a continuous process, but from the operational point of view it can be quantified to a number of short time periods $t$.

$$
\sum_{i=0}^{n} s_{i}\left(t_{k}\right)=\sum_{j=0}^{m} d_{j}\left(t_{k}\right)+L\left(t_{k}\right), t_{k} \in T
$$

where $n \in N$ is the number of active producers and $m \in M$ is the number of active consumers. The losses of power during transmission $\left(L\left(t_{k}\right)\right)$ are not considered: they are relatively small, their amount depends on network structure and their absence does not influence the theoretical solution, but allows for a simplification of the model.

Balancing is possible due to the existence of controllable devices (their operation point can be changed by the energy management system) and the ability of switching off or on a part of the consumption. In most real life installations, a microgrid is connected to an external power grid, which can provide or absorb a large amount of power. In large power grids, a constant reserve of production power is kept in order to cover occurring imbalances. Due to the high costs of this type of installation, small microgrids usually do not have such a reserve.

The presence of the power storage units, e.g. batteries, can facilitate the balancing, as they provide a time and power buffer for the management system. Power storage units are generally much faster than controllable power sources when it concerns changing the amount of taken or given energy. Extremely fast operating storage units such as flywheels can smooth the sudden peaks of power and compensate for short power loses. Large enough capacity of power storage devices in the microgrid can solve a lot of issues, even completely eliminating the imbalances. Detailed analysis of influence of power storage can be found in [5]. Storing the power unfortunately results in loses of power, a high cost of installation of storage units and, in some cases, a necessity of replacing them relatively often. In microgrids, large capacities of storage units are not common due to high costs. The most frequently considered devices are batteries, flywheels and superconductors.

The problem of balancing a microgrid is of interest to many research teams. General architectures of energy management systems might be found in [6], [7] and [8]. Details of the algorithm of the market based short-time balancing can be found in [9].

A microgrid in general can consist of producers, consumers and prosumers. Each of these can be controllable or uncontrollable. Uncontrollable devices are those which are not manageable by computer system, to this category are included most of the power consuming devices and small renewable power sources (in which power production depends on weather conditions). The balancing problem reverts to a decision problem of setting the operation point of controllable devices in the microgrid, so that supply and demand equal according to equation (1). To simplify a model, all uncontrollable devices can be aggregated to a single value: this value is either 0 (perfect balance of uncontrollable devices), positive (behaves as producer) or negative (behaves as consumer). Here, the aggregated device is assumed to be a consumer, in order to avoid a situation of overproduction by uncontrollable producers. Such situation needs special actions (e.g. removing an uncontrollable producer, wasting power, etc.), which are not common situations in the typical balancing problem.

The power sources have physical limitations: a minimal and maximal operating point, a time necessary for changing the operation point, etc. Managing a controllable power source 
means deciding if the device will be active in the next time period $\left(s_{i}\left(t_{k}\right)\right)$, and if so, determine the amount of power it will provide.

Limited information about the amount of consumption and production is a problem for balancing. The consumption is changeable in time: it is the sum of consumption of a number of small devices, where each of them may have different usage patterns. While patterns and cycles, such as daily or weekly, are usually visible in the amount of power usage, the exact amount can only be roughly predicted.

The production is the sum of the decisions of all producers which operate in the microgrid. They may or may not know how many producers are present in total and decide to participate in balancing, but in any case they can only estimate the amount of power produced by all other sources. The information shared between producers is a property of a used scheme, which can depend on the level of cooperation, the size of the microgrid or other factors, such as cost of power production, ownership, regulations, etc. In microgrids with one owner, there can be full cooperation with full flow of information; allowing for central balancing to be used. When the competition of producers is present, the flow of information may be constrained to the minimal level that is necessary for the process. When a microgrid is really small, it is helpful to know the physical limitations of the units to predict the amount of produced power.

Other factors also play important roles. When for instance the cost of operation of a power source is considered, some sources are more profitable than others. Preference to a source can be modeled by introducing the cost, which does not necessarily match the real world cost, but allows for a preference ordering that can be dynamically adjusted. Such value in market schemes is sufficient for achieving effective balancing as presented in [9]. In microgrids operating in the synchronous mode (with connection to an external power provider) the costs and profits usually dominate the decision about the production - the balancing is not the main goal of the system but becomes a constraint. In extreme cases, a certain level of imbalance might be tolerated even though it means cutting off selected consumers from the grid. Such a solution is not considered in this article. Here the microgrid is considered to be in an island mode only.

Amount of public information and what information are being exchanged is an important problem. For various reasons, as e.g. safety, competition, willingness to make profit, the producers tend to keep certain information private. The lack of information exchange can make it impossible to perform balancing. Such situation was considered by Arthur in [4], where a method to deal with such ill-defined problem is suggested. The extension of this problem, the Potluck, considers the supply and demand equalization with almost lack of information exchange.

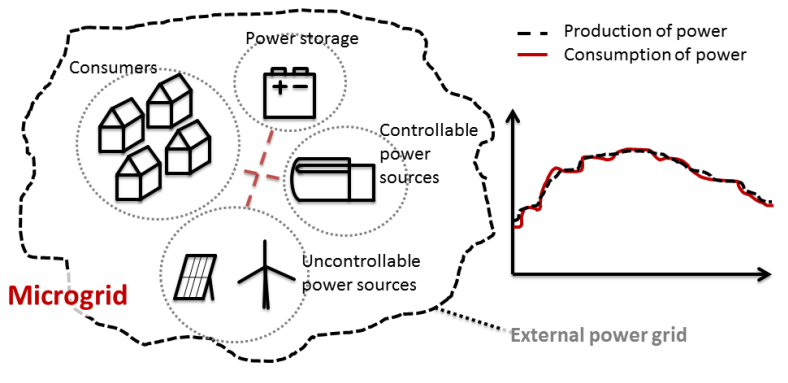

Fig. 1. Schema of the microgrid.

a) El Farol bar problem

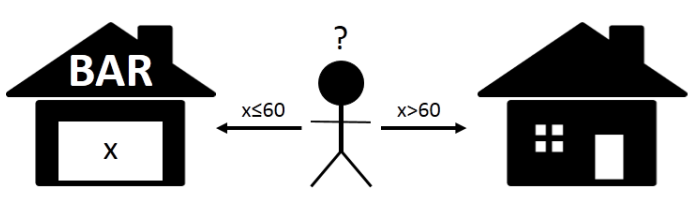

b) Potluck party problem

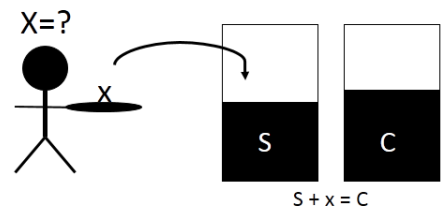

Fig. 2. Graphical representation of El Farol bar problem (a) and Potluck problem (b), $\mathrm{S}$ is a total supply and $\mathrm{C}$ is the value of consumption.

\section{El FArol AND Potluck Problem}

\section{A. Agents and communication}

To achieve common understanding of the term agent in this article, an explanation is required. In the computer science, an agent is an autonomous programmable unit that interacts with its environment and tries to fulfill given goals. Agent can be also understood as a representative of an entity, that takes decisions to achieve given goals. These two definitions are similar - both underline the autonomous decision making feature. In this article, the term agent is used in more general sense - as an entity that can make decisions. In this context, any exchange of information can be called communication, without further description on how such communication is made.

\section{B. El Farol Bar Problem}

The El Farol Bar problem (or Santa Fe Bar Problem) was introduced by Arthur in 1994 [4]. The problem was inspired by a real bar in Santa $\mathrm{Fe}$, which was very popular during Thursday nights. But if too many people decided to go to the bar to enjoy the music, it was too crowded. Arthur defined the problem as follows. If there are not more than 60 people in the bar, the people inside enjoy being there. Otherwise they feel better at home. This problem is illustrated in Fig. 2(a). So a participant is considered a winner if she/he goes to the bar while it is not crowded, or if she/he stays at home when the bar is crowded. In the El Farol Bar problem, the participants' goal 


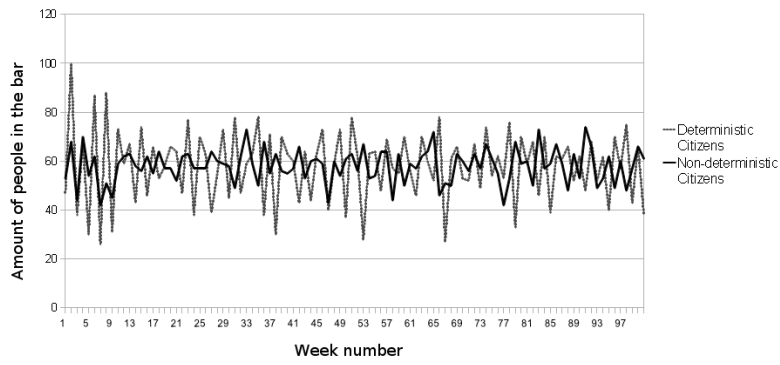

Fig. 3. Attendance to the El Farol bar of deterministic and non-deterministic citizens.

is to win as many times as possible, where the goal function $g_{i}(t)$ of $i$-th participant in the $t$-th night is defined by:

$g_{i}(t)= \begin{cases}1 & \begin{array}{l}\text { if (go to the bar and the bar not crowded) } \\ \text { or (not go and the bar is crowded ) }\end{array} \\ 0 & \begin{array}{l}\text { if (not go and the bar not crowded) } \\ \text { or (go and the bar is crowded ) }\end{array}\end{cases}$

The participants do not know how many of them are in the city, they are not communicating with each other and they have no idea what other people want to do. The only information available to them is the historic attendance: each participant knows how many people were in the bar during the last weeks. In a such defined problem there is no win-win solution - when in the bar is 60 people, the ones remaining at home loose, when there is 61 people in the bar, this 61 people loose.

In this situation, there is not enough data to make a deductive, rational decision, which makes the problem ill-defined. In [4], an inductive reasoning scheme is proposed. This is an idea taken from psychology: people are very often facing ill-defined problems and humans cope with this situation by looking for patterns and similarities in other situations. If a person would be asked the reason for going to the bar, possible answers could be: 'last week it was empty so this week it will be the same', 'last week it was full, so this week it will be empty' or just 'because I want to go'. From the game theory and mathematical analysis point of view these answers are not reasonable, but due to lack of information they are as good as any other. Humans often do not analyze all possible actions deeply, but make shortcuts and take non-optimal decisions, sometimes due to undefined reasons. It is logical from the evolution point of view, as taking decisions fast has been more crucial for survival, than being indecisive and not performing any actions. Arthur (in [4]) assumed that each person has its own way of predicting the attendance in a bar - they have a set of simple predictors. The predicted attendance might be: an average of the last few weeks; the same as last week; the same as 3 days ago (cycle detector) or assumption that the bar will always be half empty. Each person also knows the attendance from few last weeks. So a going or not-going decision depends on the known history of attendance and ones own hypothesis. What is more, participants can choose their predictors from a pool, according to the success rate of a considered predictor (how many times it gives a good advice). Surprisingly, the simulations show that the attendance in the bar is oscillating around the chosen maximal comfortable number of the participants in the bar. An example of the bar attendance in this problem is presented in Fig. 3. Arthur called it inductive reasoning method and defined it as follows. When there is a lack of knowledge to take a reasonable decision, one should use simple models that worked best in the past, and after each iteration of the process evaluate the models.

An interesting feature of this approach is that starting from some defined conditions and following totally deterministic rules, the outcome is a sequence of attendance that resembles a stochastic process. The amount of people in the bar is oscillating around 60. It is explained by the fact that citizens choose the predictors that performed best, this creates a selfregulating system where the number 60 is a natural attractor.

It is worth to notice that if the citizens know how many of them are in the city, they can solve the problem quite easy by coming to the bar in cycles. This solution was described in [10].

\section{Potluck Problem}

The Potluck problem was described and defined in [3]. A potluck is a party where every guest brings some food for everyone to eat. If the food is in an excess, the guests feel uncomfortable, as their food has to be thrown away. On the other hand, if there is a deficiency of the food, guests are hungry and unhappy. The perfect situation would be to have the exact amount of food, but the appetites of the guests depend on many factors and can vary between parties. So, without communication guests have to guess the total amount of food they have to bring, not knowing what strategy other guests will adapt, see Fig. 2(b) for an illustration.

In the Potluck problem, the goal function is to balance the supply and demand. Assuming that demand is something out of control, the goal function of $l$-th guest can be defined as, see [3]:

$$
g_{l}(t)= \begin{cases}1 & \text { if } \sum_{i=0}^{n} s_{i}(t)=\sum_{j=0}^{m} d_{j}(t) \\ 0 & \text { if } \sum_{i=0}^{n} s_{i}(t) \neq \sum_{j=0}^{m} d_{j}(t)\end{cases}
$$

The notations are explained in Table I. A guest is in the winning position when the sum of supply is equal to the sum of demand. But a guest has no means to communicate with other guests to inquire about the amount of food they plan to bring or the food they want to eat. This lack of information makes the problem ill-defined, where the rational reasoning does not help in winning of any of the guests. Enumula and Rao ([3]) define rational reasoning as applying the best strategy in given situation, that is with the assumption that the consumption level will be the same in future as in the last time. This show that it leads to increased oscillation of supply. If all guests make this assumption, they will take similar decisions, which will lead to an exaggerated change in the supply of food and the balance is never reached. The way to prevent it is by introducing different strategies for each 
of the participants, hoping that at least to some extent the undersupply and oversupply will balance.

A method to deal with this problem is presented in [3]. It is a non-rational approach similar to the inductive reasoning described in [4]. As was mentioned before, rationality, according to Enumula and Rao [3], is to apply the best strategy according to the present knowledge. In the Potluck problem, the rational action is to act as if the supply has not changed since the last party. The non-rational approach is to allow participants to take an action that is assuming a certain change in the future supply (usually not explained by analysis of the problem). Participants have a set of simple predictors with assigned weights, that forecast the level of consumption. The decision is made on the basis of a weighted sum of predictors response (weighted majority algorithm). After each party, the predictors are evaluated and weights are adjusted accordingly. Enumula and Rao [3] called it a non-rational learning algorithm. In the cited article prediction of supply side is not considered.

Enumula and Rao claim in [3] that the Potluck problem is a generalization of the El Farol Bar one. But actually the points of view of decision-makers and the goal functions are in both problems different. The personal goal function in the El Farol Bar problem is given by equation (2). It is clearly an egoistic goal, which does not consider the well-being of other participants. Decisions of a participant are influenced by the actions of others, which can be interpreted as influencing the decision-maker, but it is not done intentionally. A participant has no intention to make the bar full or not, because in both situations there is a possibility of winning. Arthur [4] underlined that participants are independent agents, that are following their goals, even not being aware how many of participants are in the system.

In the Potluck problem, the goal function is to balance supply and demand, as described by equation (3). The goal can be defined as a global goal function which means that it is a type of a social welfare function. Unlike the El Farol Bar problem, it does not consider a personal gain or loss, but the sum: all participants win or lose. In the El Farol problem, if the bar is crowded, the people in the bar loose, but the people that did not go to the bar win. An analogy to social welfare in the El Farol Bar problem would be the situation where the citizens are trying to reach 60 people in the bar every week, and in case when there are more or less attendees everyone looses.

The question arises if these problems are really equivalent although the goals of the participants are different. The methods of approaching them are similar, but the problems complexity is changing in them when some exchange of information is introduced. In case of a personal goal, adding knowledge about the decisions of others (by communication) only introduces complications in decision making: the agent has to actively make effort to be in the winning position. To clarify this statement a following scenario can be considered: there are 100 participants in the El Farol bar problem, but just 99 of them has some media of communicating their decisions, e.g. announcing it on the social network. None of them knows

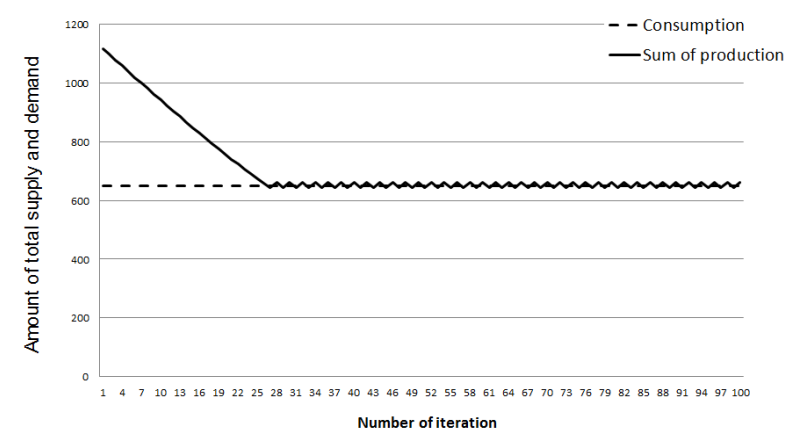

Fig. 4. Potluck problem simulation with linear consumption, with $n=100$.

what the 100th participant will decide, and this participant does not know the decisions of others. By communicating each other, the participants can agree to perform a schema that will ensure fair amount of winnings for each agent. They can agree that 59 of them are going to the bar and 40 staying home (the agents that are going to the bar can change every week, which would mean introducing going to the bar in cycles). This is a solution where the winner group is the largest, independent of the decision made by the isolated person. But it requires of participants to make concessions for some kind of social fairness. In the original problem agents are assumed to be myopic and egoistic, which does not allow them to cooperate. So, seeing the situation, participants staying home will be willing to change their decision. If this happens, the situation will change again and the decisions of the participant will also change. That would trigger a set of changes that would lead to an apparently chaotic behavior. A stopping condition may be applied, e.g. it might be the time (an hour of going to the bar is defined) or the number of decision changes. When the decision making process is closed the number of citizens in the bar is very likely to be not optimal. The outcome will show pseudo stochastic oscillations around the number of 60 people going to the bar, even when almost everything is known. In the Potluck problem the behavior in this scenario is different. Information about the amount of food brought by 99 out of 100 people suggest their predicted consumption level and all participants try to minimize the error of prediction. After a number of iteration the 99 participants can predict the production level of the 100th participant and consider his decision. Imbalance is still present, but the oscillations are relatively smaller. Socially aware agents are more likely to cooperate, make concessions and negotiate their decisions. Introduction of communication to the problem makes it possible to reason rationally.

\section{FROM POTLUCK TO POWER BALANCING}

Table I presents a comparison of the theoretical El Farol Bar problem, the Potluck problem and a practical problem of power balancing. The problems seem very similar, but a quick analysis shows main differences which cause that distinct methods of facing these problems have to be considered. The theoretical problems are very simplified and constrained. The 
TABLE I

COMPARISON BETWEEN PROBLEMS OF El FAROL, POTLUCK AND POWER BALANCING.

\begin{tabular}{|l|l|l|l|}
\hline Symbol & El Farol Problem & Potluck Problem & Energy balancing \\
\hline \hline$T$ & set of weeks & set of weeks & set of time periods \\
\hline$N$ & set of participants & set of guests & set of suppliers \\
\hline$M$ & equal to 1 & set of consumers & set of energy consumers \\
\hline$s_{i}(t)$ & binary decision of going or not & $\begin{array}{l}\text { amount of brought food by } i \text {-th } \\
\text { guest }\end{array}$ & $\begin{array}{l}\text { amount of energy produced by } i \text {-th } \\
\text { supplier }\end{array}$ \\
\hline$d_{j}(t)$ & constant & $\begin{array}{l}\text { amount of food expected by con- } \\
\text { sumer } j\end{array}$ & $\begin{array}{l}\text { amount of energy demanded by } \\
\text { consumer } j \text { in time } t\end{array}$ \\
\hline$S(t)=\sum_{i=0}^{N} s_{i}(t)$ & $\begin{array}{l}\text { total attendance in the bar in a } \\
\text { week } t\end{array}$ & $\begin{array}{l}\text { amount of food brought to the party } \\
\text { amount of energy produced by all } \\
\text { suppliers }\end{array}$ \\
\hline$D(t)=\sum_{j=0}^{M} d_{j}(t)$ & constant & total demand of food & total consumption \\
\hline$P_{i}(t)$ & $\begin{array}{l}\text { prediction of the attendance to the } \\
\text { bar in a week } t\end{array}$ & $\begin{array}{l}\text { prediction of the amount of total } \\
\text { consumption in a week } t\end{array}$ & $\begin{array}{l}\text { prediction of total consumption in } \\
\text { time } t\end{array}$ \\
\hline
\end{tabular}

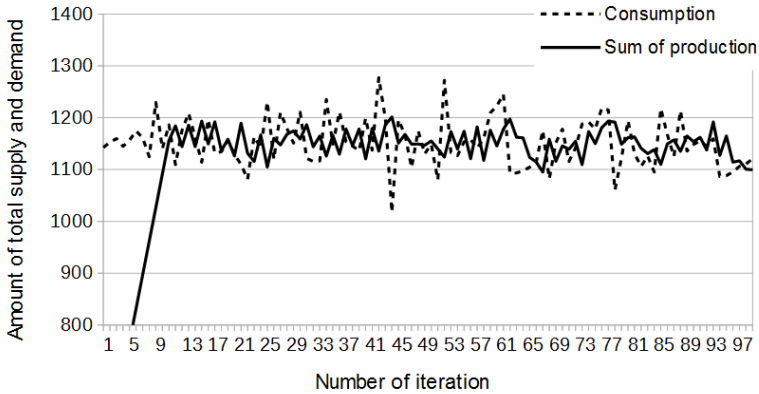

Fig. 5. Potluck problem simulation with random consumption, with $n=100$.

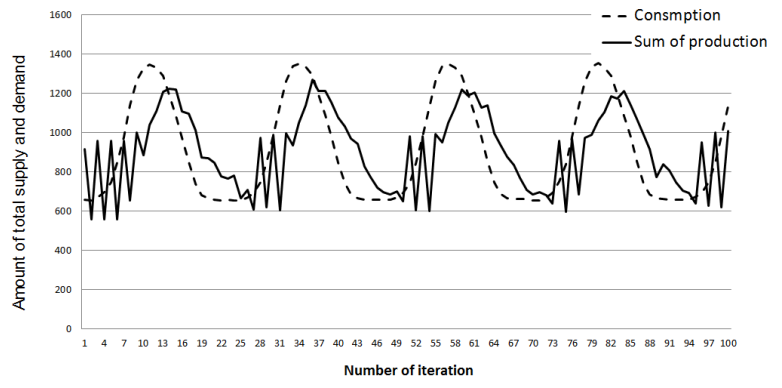

Fig. 6. Potluck problem simulation with sinusoidal consumption, with $\mathrm{n}=100$.

most limiting constraint is that agents are banned from exchanging information. The Potluck problem can be expanded with additional constraints that resemble physical limitations that are present in the power balancing problem (e.g. minimal operating point, maximal operating point, latency of operating point change, etc.), but these do not significantly change the problem considered: it is still an ill-defined decision problem, the additional constraints do not simplify nor complicate it.

In the Potluck problem, lack of information about power production is equally problematic as lack of knowledge about its consumption. Tests have been made using a non-rational learning algorithm with different consumption patterns: the performance of the algorithm with a random consumption is shown in Fig. 5, with a fast changing sinusoid consumption in Fig. 6 and with a linear consumption in Fig. 4. Several categories of predictors have been used in the calculations:

- average demand over the last $k$ periods,

- randomly chosen value of demand from the last $k$ periods,

- choosing the demand from $t-k$ period, this predictors are cycle detector, it can detect cycles of 2, 3, 5 periods,

- mirror image around the average of the last $k$ periods,

- the same as the previous period,

- trend over the last $k$ periods,

- median of the last $\mathrm{k}$ periods,

- weighted solution over the last $\mathrm{k}$ periods - the random $\mathrm{k}$ weights are chosen: $w_{1}, w_{2}, \ldots, w_{k}$, where $\sum_{i=1}^{k} w_{i}=1$ and the prediction is calculated as: $\sum_{i=1}^{k} D(t-i) w_{i}$.

- the smallest value of demand out of the two last periods,

- the larger value of demand out of the two last periods.

It is clear that a less changeable consumption makes it easier to reduce imbalances, as predictors work better. However, even for linear consumption agents could not fully balance demand and supply. The oscillations are still visible.

The reason for this is that suppliers use the same algorithm and therefore take similar decisions based on the same information, which in turn leads to overcompensation. This is logical, as a supplier has no knowledge of other suppliers and tries to solve the imbalance by itself. In some situations (e.g. the oscillations in the linear case) the result can be improved by introducing additional conditions to the agents' logic, but such specialization would decrease overall performance in the general case. A better solution is to allow for communication between the suppliers.

There are many ways in such communication can be introduced. The simplest case considers publishing information for all agents. One way to prevent big oscillations is to limit the number of suppliers that can change their decision, such that not all suppliers will react to the imbalance. This automatically limits the total change. It can be achieved by introducing tokens to tell a supplier that it is allowed to change its output, and publishing which agents have the tokens in the given iteration. Preferring certain suppliers over others becomes a 
matter of a central body that has to decide how the tokens are distributed. Another way of preventing big oscillations is by introducing direct communication between the suppliers. This can lead to bilateral and multilateral negotiations, which permit for rational reasoning. In a similar way, a solution with an ordering of suppliers can be introduced, and the agents higher in hierarchy would be privileged to change their supply. In both last approaches, preference of a supplier can be decided by all the suppliers, using the information they share, without a central decision body.

Realistically, a rotation of the suppliers should be introduced, based on various factors, such as e.g. the cost of supply. To dynamically adjust the ordering, a market scheme can be adopted: prices will introduce a certain order. Exchanging information about the price and defining a cost of imbalance (the bigger the difference between supply and demand the higher the cost) is a simple market based scheme for balancing. Considering such approach requires concessions from participants, but also allows for rational decision making and leads to almost perfect balancing.

The goal function in real life power balancing is much more complex than in the artificial, theoretical problems. Comparing the goal functions (equations (1) and (3)) can give impression that they are the same. But in many of the described models the criterion is to maximize the profit or minimize the cost of producing energy ([9], [11]), where achieving balance is just one of the conditions. Often only these microgrids are considered that have connection to the external power network. Such a reserve (external network is not a constraining factor in this case: it can supply or receive any amount of power) is ensuring that the balance can always be achieved, which facilitates the decision making. In such conditions, the problem of balance is not the primary one and the goal function focus usually on profitability of the power production. The concept of a microgrid is fairly new. Pointing out that it can generate a revenue can motivate further development of this technology and construction of microgrids. When the island mode operation of the microgrid is considered, the power balancing becomes crucial for safety of the devices and the network itself.

In the Potluck and the El Farol problems decisions must be taken in discrete time intervals and need many iterations to allow the learning algorithms to adjust the predictors. After each iteration the outcome is calculated - the amount of people in the bar or amount of food on the party. The power balancing problem is in reality a continuous process, but it is often quantified to allow computer algorithms to cope with. The shorter the quantification time, the more small changes can be balanced, leading to smaller loses and better security of the grid. However, shortening of the balancing time has also its limits; as the change of the operation point of the devices requires time. Different devices have varied times of reactivity regarding their operation point change, which makes it impossible to derive the optimal minimal length of a time period in general. It can be approximated when the real set of devices that are installed in the defined microgrid is known.
At present, for energy management system, the time periods may be 10 minutes, 5 minutes, but seldom less than 1 minute. Of course, the minimal physical time depends on the set of devices, but that can be evaluated only experimentally.

\section{METHODS OF POWER BALANCING}

The problem of power balancing is slightly different on each level of the power grid. Balancing power in the high voltage network can benefit from big aggregation of consumption. There the daily and weekly cycles dominate [12] and the inertia of the grid is much larger. In microgrids, the changes in consumptions still have visible cycles, but the random behavior plays a bigger role and the inertia of devices is smaller. This requires fast decision making regarding changing the operation point of sources and consumers in the grid. That poses a computation challenge, especially when the number of nodes is large and an energy management system has to balance the energy in all nodes, considering also all the physical limitation of the devices within a defined time period.

Effective balancing requires some kind of communication or a schema of cooperation between the producers of energy. The most straightforward schema is the centralized management: it is then possible to have one predictor of demand (e.g. that which gives the smallest errors), based on which the plan for production is made and the system distributes the power production. Centralized systems offer possibility of optimal production distribution [11], possibly considering multicriteria decision making. Centralized systems unfortunately have a number of different disadvantages: sensitivity to central controller failure, poor scalability, and requirement of full control over the sources. Full control may not be a problem in microgrids with a single owner, but may be unacceptable in a general situation. A centralized system might also not be able to consider specific preferences of the source owners or might give unacceptable results when a source owner happens to actively make decisions on its own (although that should not happen in a well designed system).

Non-centralized solutions have been also developed and showed promising results. Agent-based power balancing systems are quite popular approach. Due to the intrinsic characteristics of the agents, these system are distributed. A classification of different energy management schemes for agent-based systems can be found in [13]. Agents can represent single devices, nodes in the power grid, subsets of nodes or even single microgrids. The presented categories of management schemes are central-hierarchical control structure, distributedhierarchical control structure, and decentralized control structure (peer-to-peer relation). The hierarchical organization of agents introduces an order and defines agent's functions in optimization and decision making. This can speed up the processing of the data, by dividing and distributing the tasks for calculation. The hierarchy can handle power distribution in a similar way as centralized systems. Completely decentralized control structures are extremely robust to failures and can quickly adapt to changing conditions, but because there is a larger exchange of data and negotiation, such systems tend 
to operate slower, which might be the source of additional imbalances.

The last group of control systems are the ones based on market structures. The market is the central element of the balancing process, but the participants decide what kind of offer is placed on the market. In such approaches, money and cost functions play the role of ordering the power from most desired sources (i.e. cheapest and most efficient) down to the sources that are used only in emergency (i.e. more expensive power systems). Presentation of market based energy control systems can be found in [6], [9], [14].

\section{CONCLUSION}

The power balancing problem in microgrids is especially important when the island mode operation is considered.

There are many methods that approach this problem with success, by using well known concepts such as markets. However, question arises whether other and perhaps simpler schemes might also be successful and more performent. This problem is adressed in the paper.

Practical power balancing has a lot of different limitations, which are very difficult to model: profitability, physical limitations, long term contracts for power, interaction between sources, delays in changing operation point, etc. Despite of this, theoretical models of the grid can give many useful information about the behavior of the real world system in an extremely controlled environment. The Potluck problem is very simplistic, but it touches the core of balancing: distributed decision making, in a situation of insufficient knowledge about demand and supply. Unfortunately, the problem is ill-defined and there are simply not sufficient data to create a rationallearning algorithm. The inductive learning and non-rational approaches give results that are oscillating around the ideal solution which is acceptable for theoretical problems but is unacceptable in power balancing.

In the Potluck problem, it is clear that the uncertainty is on both supply and demand side. The power balancing problem is different, as the demand can be predicted and a quick adjustment of the supply can be done in order to compensate the errors of the prediction. The inertia of the devices defines a time in which this adjustment has to be made: the faster the managing algorithm is, the smaller are the imbalances that occur, and the better it is for the grid devices.

The Potluck problem further shows that, when demand is constant and known to the suppliers, autonomous decisions made by the suppliers can actually result in a big oscillation of supply. Having the same set of information (even true and exact) cannot help if all suppliers are taking rational decisions, but do not consider decisions of other suppliers.

This also applies to the power balancing. Certain willingness to cooperate and to make concessions is needed to assure that the balancing works, with minimal oscillations. Reactiveness to the current situation and to other participants turns out to be more important than historic data about power usage and production. As shown in the experiments, knowledge about behavior of other producers is more important than historical knowledge of consumers. More accurate predictions help to improve the situation, but as human behavior is quite unpredictable, not much improvement can be made. The Potluck problem can be solved with a rational solution, if certain constraints are relaxed. Relaxing the constraint pertaining information sharing between suppliers, which we consider to be in a form of communication, is sufficient. In this situation, the Potluck problem closer resembles the power balancing one, where communication or sharing of information between producers is also sufficient to achieve a balancing within the time in which the devices need to react.

Moving from traditional grids to microgrids in the island mode is a huge modification and poses new technological challenges, not only in the physical structure, but also in the management and underlying schemes. Communication between controllable producers is anyway becoming a standard in modern power grids. This evolution gives enough information to develop a management scheme for a microgrid in the island mode, where proper balancing can be achieved within the limits of available production and consumption.

\section{ACKNOWLEDGMENT}

The research of W. Radziszewska was supported by the Foundation for Polish Science under International $\mathrm{PhD}$ Projects in Intelligent Computing. Project financed from The European Union within the Innovative Economy Operational Programme 2007-2013 and European Regional Development Fund.

\section{REFERENCES}

[1] R. Palma-Behnke, C. Benavides, E. Aranda, J. Llanos, and D. Saez, "Energy management system for a renewable based microgrid with a demand side management mechanism," in Computational Intelligence Applications In Smart Grid (CIASG), 2011 IEEE Symposium on. IEEE 2011, pp. 1-8.

[2] V. Balijepalli, V. Pradhan, S. Khaparde, and R. M. Shereef, "Review of demand response under smart grid paradigm," in Innovative Smart Grid Technologies - India (ISGT India), 2011 IEEE PES, Dec 2011, pp 236-243.

[3] P. K. Enumula and S. Rao, "The potluck problem," CoRR, vol. abs/0809.2136, 2008.

[4] W. B. Arthur, "Complexity in economic theory: Inductive reasoning an bounded rationality," The American Economic Review, vol. 84, no. 2, pp. 406-411, May 1994.

[5] P. Vytelingum, T. D. Voice, S. D. Ramchurn, A. Rogers, and N. R. Jennings, "Theoretical and practical foundations of large-scale agentbased micro-storage in the smart grid," J. Artif. Int. Res., vol. 42, no. 1 , pp. 765-813, Sep. 2011.

[6] H. Vogt, H. Weiss, P. Spiess, and A. Karduck, "Market-based prosumer participation in the smart grid," in 4th IEEE International Conference on Digital Ecosystems and Technologies (DEST). IEEE, 2010, pp. 592-597.

[7] W. Radziszewska, Z. Nahorski, M. Parol, and P. Pałka, "Intelligent computations in an agent-based prosumer-type electric microgrid control system," in Issues and Challenges of Intelligent Systems and Computational Intelligence, ser. Studies in Computational Intelligence, L. T. Kóczy, C. R. Pozna, and J. Kacprzyk, Eds. Springer International Publishing, 2014, vol. 530, pp. 293-312. [Online]. Available: http://dx.doi.org/10.1007/978-3-319-03206-1_20

[8] S. Ramchurn, P. Vytelingum, A. Rogers, and N. Jennings, "Putting the 'smarts' into the smart grid: a grand challenge for artifitial intelligence," Communications of ACM, vol. 55, no. 4, pp. 86-97, 2012.

[9] P. Pałka, W. Radziszewska, and Z. Nahorski, "Balancing electric power in a microgrid via programmable agents auctions," Control and Cybernetics, vol. 4, no. 41, pp. 777-797, 2012. 
[10] J. R. Leady, "If nobody is going there anymore because it's too crowded, then who is going? experimental evidence of learning and imitation in the el farol coordination game," august 2007. [Online]. Available: http: //ssrn.com/abstract=1275537orhttp://dx.doi.org/10.2139/ssrn. 1275537

[11] A. Tsikalakis and N. Hatziargyriou, "Centralized control for optimizing microgrids operation," Energy Conversion, IEEE Transactions on, vol. 23, no. 1, pp. 241-248, March 2008.

[12] A. Lovins, M. Odum, J. Rowe, and J. Rowe, Reinventing Fire: Bold Business Solutions for the New Energy Era. Chelsea Green Publishing Company, 2011. [Online]. Available: http://books.google.com.au/books?

\section{id=ZQVZxsGFjnAC}

[13] G. Rohbogner, S. Fey, U. Hahnel, P. Benoit, and B. Wille-Haussmann, "What the term agent stands for in the smart grid definition of agents and multi-agent systems from an engineer's perspective," in Computer Science and Information Systems (FedCSIS), 2012 Federated Conference on, Sept 2012, pp. 1301-1305.

[14] M. Vasirani and S. Ossowski, "A collaborative model for participatory load management in the smart grid," in Proc. 1st Intnl. Conf. on Agreement Technologies. CEUR, 2012, pp. 57-70. 